\title{
Aspectos clínico-hematológicos e lesões vesicais na intoxicação crônica espontânea por Pteridium aquilinum em bovinos ${ }^{1}$
}

\author{
Adriane L. Gabriel ${ }^{2}$, Glaucia D. Kommers ${ }^{3}$, Eduardo K. Masuda ${ }^{2}$, Rafael A. Fighera ${ }^{3}$, \\ José V. M. Piazer ${ }^{4}$, Claudio S.L. Barros ${ }^{3}$, Tessie B. Martins ${ }^{5}$ e Fábio B. Rosa ${ }^{5}$
}

\begin{abstract}
Gabriel A.L., Kommers G.D., Masuda E.K, Fighera R.A., Piazer J.V.M., Barros C.S.L., Martins T.B. \& Rosa F.B. 2009. [Clinical and hematological aspects and urinary bladder lesions in chronic spontaneous poisoning by Pteridium aquilinum in cattle.] Aspectos clínicos-hematológicos e lesões vesicais nas formas crônicas de intoxicação espontânea por Pteridium aquilinum em bovinos. Pesquisa Veterinária Brasileira 29(7):515-525. Departamento de Patologia, Universidade Federal de Santa Maria, Camobi, Santa Maria, RS 97105-900, Brazil. E-mail: glaukommers@yahoo.com

Spontaneous cases of chronic poisoning by Pteridium aquilinum in cattle were studied. The clinical forms of the disease were squamous cell carcinoma (SCCs) of the upper digestive tract (UDT) and bovine enzootic hematuria (BEH). The cases were from the midland Region of the Midwest of Rio Grande do Sul State, Brazil, and were submitted to the Laboratório de Patologia Veterinária of the Universidade Federal de Santa Maria. Clinical signs and blood work were evaluated at terminal phase of disease. Cattle with UDT SCCs had progressive weigth loss, ruminal atony, cough, dysphagia, bloating, and regurgitation. In cattle with $\mathrm{BEH}$, hematuria was observed in all cases, followed by progressive weight loss. Non-regenerative anemia was detected in $33.33 \%$ of the cattle with UDT SCCs form and in $66.66 \%$ of the cattle with BEH form. Changes in white blood count occurred in some cases but drop in lymphocyte numbers was uncommon in both forms of disease. For the morphological study, urinary bladders from 46 cattle with UDT SCCs and 11 cattle with BEH were analyzed. Grossly, 16/46 bladders from the UDT SCCs form had gross lesions (red or pale vesical nodules, hemorrhages, and papilomas; red urine was detected at necropsy of only three cases). In BEH form, the bladder had nodules, large neoplastic masses, red urine, papilomas, and hemorrhages. Pyelonephritis and hydronephrosis were seen in a few cases. Microscopically, in the UDT SCCs form, $44 / 46(95.65 \%)$ bladders had 22 different types of morphological changes, characterized by neoplastic lesions (5/22) and non-neoplastic lesions (17/22); the latter were subdivided in non-neoplastic epithelial changes (6/17), general changes of the lamina propria (6/ 17 ), and inflammatory changes (5/17). The bladder changes in $\mathrm{BEH}$ form were of 19 different types, characterized by neoplastic lesions (5/19) and non-neoplastic lesions $(14 / 19)$, which were subdivided in non-neoplastic epitelial changes $(9 / 14)$, general changes of the lamina propria (3/14), and inflammatory changes (2/14). In $\mathrm{BEH}$, mesenchymal neoplasms were more observed than epithelial ones, and most of them were malignant.
\end{abstract}

\footnotetext{
${ }^{1}$ Recebido em 24 de dezembro de 2008.

Aceito para publicação em 26 de fevereiro de 2009.

Parte da Dissertação de Mestrado do primeiro autor (Pesquisador bolsista do CNPq - Conselho Nacional de Desenvolvimento Científico e Tecnológico).

2 Programa de Pós-Graduação em Medicina Veterinária, área de concentração em Patologia Veterinária, Centro de Ciências Rurais
} 
Immunohistochemistry was utilized to characterize the histogenesis of poorly differentiated neoplasms. In conclusion, the morphological study demonstrated that urinary bladder lesions identical to the those seen in cattle with $\mathrm{BEH}$, are very often present in cattle with the UDT SCCs form.

INDEX TERMS: Diseases of cattle, poisonous plants; Pteridium aquilinum, bovine enzootic hematuria, squamous cell carcinomas of the digestive system, veterinary pathology.

RESUMO.- Foram estudados casos espontâneos de intoxicação crônica por samambaia (Pteridium aquilinum) em bovinos nas formas clinicopatológicas de carcinoma de células escamosas (CCE) no trato alimentar superior (TAS) e de hematúria enzoótica bovina (HEB), provenientes da Mesorregião Centro Ocidental Rio-Grandense e encaminhados ao Laboratório de Patologia Veterinária da Universidade Federal de Santa Maria. Para o estudo clínico foram avaliados os sinais clínicos de bovinos com CCEs no TAS e com HEB e realizados hemogramas na fase terminal da doença. Os principais sinais clínicos nos bovinos com CCEs no TAS foram emagrecimento progressivo, atonia ruminal, tosse, disfagia, timpanismo e regurgitação. Nos bovinos com HEB, hematúria foi o principal sinal, observado em todos os casos, seguido de emagrecimento progressivo. No exame hematológico, 33,33\% dos bovinos com CCEs no TAS e $66,67 \%$ dos bovinos com HEB apresentaram anemia arregenerativa. Alterações no leucograma ocorreram em alguns casos, mas linfopenia foi um achado infreqüente em ambas as formas de intoxicação. Para o estudo morfológico, foram avaliadas as bexigas de 46 bovinos com CCEs no TAS e de 11 bovinos com HEB. Macroscopicamente, 16/46 bexigas dos casos de CCEs no TAS apresentaram alterações macroscópicas, que consistiam nódulos vermelhos ou pálidos, hemorragia e papilomas; urina vermelha foi observada na necropsia de apenas três casos). Nos casos de HEB, os achados macroscópicos vesicais foram nódulos vermelhos, massas neoplásicas focalmente extensas, urina vermelha, papilomas, hemorragias e ruptura de bexiga; pielonefrite e hidronefrose foram observados em poucos casos. Histologicamente, 44/46 (95,65\%) bexigas de bovinos com CCEs no TAS apresentaram 22 tipos diferentes de alterações morfológicas, que foram classificadas em alterações neoplásicas (5/22) e alterações não-neoplásicas (17/22); essas últimas foram divididas em alterações epiteliais nãoneoplásicas (6/17), alterações gerais na lâmina própria (6/ 22) e alterações inflamatórias (5/17). Os achados histológicos das bexigas dos casos de HEB foram classificados da mesma forma, resultando em 19 tipos diferentes de alterações morfológicas. Dessas, 5/19 eram alterações neoplásicas e 14/20, alterações não-neoplásicas (9/14 alterações epiteliais não neoplásicas, $3 / 14$ alterações gerais na lâmina própria e 2/14 alterações inflamatórias). Na HEB, os neoplasmas mesenquimais foram mais freqüentemente observados que os epiteliais, e a maior parte era maligna. A técnica de imuno-histoquímica foi utilizada para caracterizar os aspectos morfológicos, principalmente dos neoplasmas. Através do estudo morfológico concluiu-se que é muito freqüente a ocorrência de lesões vesicais em bovi- nos com a forma crônica de CCEs no TAS e que essas lesões são idênticas às encontradas nos bovinos com HEB.

TERMOS DE INDEXAÇÃO: Doenças de bovinos, plantas tóxicas, Pteridium aquilinum, hematúria enzoótica bovina, carcinoma de células escamosas do trato digestório, patologia veterinária.

\section{INTRODUÇÃO}

A samambaia, Pteridium aquilinum, é uma planta de grande importância e ampla distribuição que causa diversos quadros clínicos de intoxicação em animais domésticos. Em bovinos causa três quadros clínicos de intoxicação, um agudo e dois crônicos. Nas formas crônicas estão incluídos os carcinomas de células escamosas (CCEs) no trato alimentar superior (TAS) e a hematúria enzoótica bovina (HEB) caracterizada por neoplasmas vesicais (Tokarnia et al. 2000). Ambas as formas são responsáveis por grandes perdas econômicas em regiões endêmicas (Riet-Correa \& Medeiros 2001), porém a prevalência dessas formas varia de região para região (Tokarnia et al. 1969).

A concentração dos princípios tóxicos pode variar dependendo de condições geográficas e ambientais (Hirono et al. 1973, Villalobos Salazar et al. 1999) e pode talvez influenciar na ocorrência de uma ou de outra forma crônica da intoxicação (Gava et al. 2002). A idade dos bovinos, a quantidade de planta ingerida e o tempo de ingestão também estão diretamente relacionados ao tipo de intoxicação observada (Döbereiner et al. 1967).

A coexistência de lesões vesicais e no trato alimentar nas duas formas crônicas da intoxicação tem sido descrita em bovinos e, nesses casos, a lesão mais acentuada, aquela que finalmente causa o aparecimento dos sinais clínicos mais evidentes, determina a forma clínica apresentada pelo animal (Döbereiner et al. 1967, Tokarnia et al. 1969).

Nos últimos anos, estudos das formas crônicas de intoxicação espontânea por $P$. aquilinum têm sido realizados no Laboratório de Patologia Veterinária (LPV) da UFSM (Souto 2006a,b, Masuda 2007). Nesses trabalhos foram enfatizadas as alterações características de uma ou de outra forma clínica crônica. Entretanto, a sobreposição das lesões nas duas formas, especialmente a avaliação das lesões vesicais nos casos da forma crônica de CCEs no TAS, não tinha sido abordada nesses estudos.

Os objetivos gerais deste estudo foram avaliar parâmetros clínico-hematológicos e morfológicos das formas crônicas de intoxicação por $P$. aquilinum. Os aspectos clínico-hematológicos foram avaliados pelos sinais clíni- 
cos e as alterações hematológicas na fase terminal da doença. Para o estudo morfológico foram estudadas as lesões vesicais observadas em ambas às formas de intoxicação crônica.

\section{MATERIAL E MÉTODOS}

Foram estudados casos espontâneos de intoxicação crônica pela samambaia, Pteridium aquilinum (L.) Kuhn, em bovinos, nas formas clínicas de carcinoma de células escamosas (CCEs) no trato alimentar superior (TAS) e de hematúria enzoótica bovina (HEB), provenientes de municípios da Mesorregião Centro Ocidental Rio-Grandense encaminhados ao Laboratório de Patologia Veterinária da Universidade Federal de Santa Maria (LPV-UFSM) entre 2003 e 2008.

Para o estudo clínico-hematológico foram avaliados os sinais clínicos observados em 30 bovinos com CCEs no TAS, nove com HEB confirmada pela necropsia e nove com HEB clínica (consideraram-se aqui nove bovinos que apresentaram hematúria e provenientes de propriedades rurais infestadas pela planta nas áreas de pastoreio). Para avaliar o perfil hematológico na fase terminal de ambas as formas crônicas, foram realizados hemogramas de 30 bovinos com CCEs no TAS, seis com HEB confirmada pela necropsia e nove com HEB clínica. Amostras de sangue, coletadas durante o atendimento clínico dos animais com suspeita de intoxicação crônica por $P$. aquilinum foram remetidas e os hemogramas realizados conforme técnicas convencionais. Os valores de referência para os exames hematológicos foram determinados de acordo com Jain (1986). Quando necessário, foi realizado o exame do sedimento da urina, conforme técnicas padrões.

Os bovinos afetados tiveram morte espontânea ou foram submetidos à eutanásia devido à deterioração do quadro clínico. Foi realizada a necropsia e os materiais coletados. Para o estudo morfológico, foram avaliadas macroscopicamente e histologicamente as bexigas de 11 casos de HEB e de e 46 casos de CCEs no TAS nos quais a bexiga (com ou sem alterações macroscópicas) foi remetida junto com os outros órgãos. Bexigas normais de 10 bovinos foram utilizadas como controles. Após a fixação em formol $10 \%$, os materiais foram encaminhados ao processamento histológico de rotina. Foram clivados fragmentos de todas as lesões macroscopicamente visíveis e dois fragmentos de regiões aleatórias nos casos em que não havia lesão vesical aparente. Cortes de $3 \mu \mathrm{m}$ foram utilizados para as avaliações histológica e histoquímica (ácido periódico de Schiff [PAS] e azul alciano).

O detalhamento das lesões do TAS de grande parte dos bovinos incluídos neste trabalho foi abordado em outros dois estudos (Souto et al. 2006a, Masuda 2007).

As lesões da bexiga foram classificadas em: a) neoplásicas (epiteliais ou mesenquimais; malignas ou benignas); b) nãoneoplásicas (anormalidades epiteliais não-neoplásicas [hiperplasia, displasia, ninhos de Brunn, cistite polipóide, cistite cística, cistite glandular, metaplasia glandular, metaplasia escamosa, vacúolos intraepiteliais], alterações gerais na lâmina própria [dilatação de vasos linfáticos, hemorragia, edema, estroma mixóide, tecido fibrovascular, tumefação de células endoteliais] e somente inflamatórias [infiltrado inflamatório, folículos linfóides, cistite fibrinossupurativa, cistite crônica, mastócitos na lâmina própria]. Esta classificação foi feita de acordo com critérios descritos anteriormente (Peixoto et al. 2003, Murphy et al. 2004, Sardon et al. 2005, Carvalho et al. 2006).
Em cortes selecionados foi realizada a técnica de imunohistoquímica em lâminas silanizadas com seções histológicas de $3 \mu \mathrm{m}$. Foram utilizados os seguintes anticorpos (Acs) primários: Ac policlonal anti-pancitoceratina bovina produzido em coelho, (DakoCytomation, cód. Z0622), na diluição 1:4000; Ac monoclonal anti-vimentina suína, clone V9 (DakoCytomation, cód. M0725), na diluição 1:100, e Ac policlonal anti-fator de von Willebrand humano, (DakoCytomation, cód. A0082), na diluição 1:400. Foi utilizada a técnica do complexo estreptavidina-biotinaperoxidase (LSAB Kit Peroxidase Universal, DakoCytomation, cód. K0690).

\section{Sinais clínicos}

\section{RESULTADOS}

Os sinais clínicos observados nos 30 bovinos com CCEs no TAS, em ordem decrescente de freqüência foram: emagrecimento progressivo (29/30), atonia ruminal (22/30), tosse $(20 / 30)$, disfagia $(18 / 30)$, timpanismo $(9 / 30)$, regurgitação $(9 / 30)$, estertor $(6 / 30)$, apetite seletivo $(6 / 30)$, sialorréia $(6 / 30)$, anorexia $(6 / 30)$, extensão do pescoço $(5 / 30)$, fraqueza $(5 / 30)$, dificuldade de deglutir (4/30), halitose $(3 / 30)$, decúbito (2/30), dispnéia (2/30), edema de faringe (2/30), secreção nasal (1/30) e queda na produção de leite (1/30). Freqüentemente eram observados grumos de alimento pouco digerido nas fezes. Um dos bovinos apresentou sinais clínicos de ambas as formas crônicas de intoxicação, caracterizados por emagrecimento progressivo, tosse (incluídos na contagem acima) e hematúria.

Os sinais clínicos descritos nos bovinos com HEB confirmada pela necropsia (9 animais) e nos casos clínicos (9 animais) foram, em ordem decrescente de freqüência, hematúria (18/18; com o tempo de duração informado em três casos de 40, 90 e 180 dias e intermitente em outro), emagrecimento progressivo (9/18), mucosas pálidas (4/ $18)$, apetite seletivo $(2 / 18)$, anorexia (2/18), decúbito lateral (1/18), dor abdominal (1/18), polaciúria (1/18), prostração (1/18), fraqueza (1/18) e tenesmo (1/18). Em um dos casos foi detectada, por palpação retal, uma massa no interior da cavidade pélvica.

\section{Hematologia}

Foram realizados 30 hemogramas de bovinos com lesões no TAS. Desses, 20 não apresentaram alterações no eritrograma e 10 apresentaram anemia arregenerativa leve. Nos animais anêmicos, o número de eritrócitos variou de 3,4 a $4,9 \times 10^{6} / \mathrm{mm}^{3}$ de sangue (valores de referência - VR: 5,0-10,0 x 10\%/ $\mathrm{mm}^{3}$ ); o hematócrito de 1823\% (VR: $24-46 \%$ ) e o volume corpuscular médio (VCM) variou de 46,9 a 56,2 fl (VR: 40,0-60,0 fl). Vinte e quatro bovinos não apresentaram alterações no leucograma. Seis apresentaram desvio à esquerda, cinco deles regenerativos e um degenerativo. Concomitantemente ao desvio a esquerda, um bovino apresentou linfopenia, outro eosinofilia, e um outro monocitose.

Foram realizados 15 hemogramas de bovinos com HEB e em seis deles as lesões vesicais foram confirmadas pela necropsia. Desses 15 bovinos, cinco não demonstraram alterações no eritrograma e 10 apresentaram anemia 

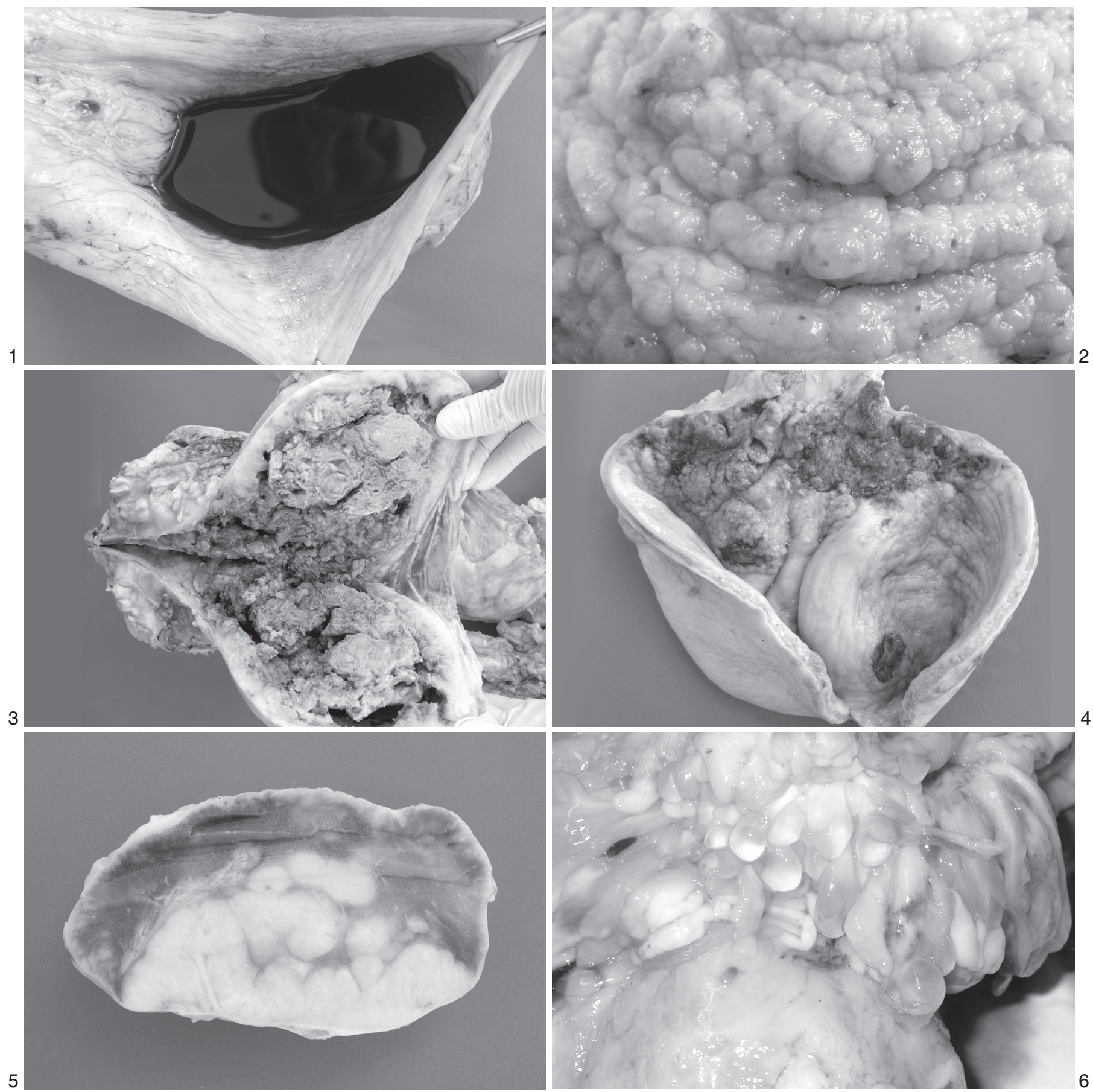

Fig.1. Urina vermelho-escura na bexiga de bovino na forma clínica de carcinoma de células escamosas no trato alimentar superior. Observam-se também nódulos e hemorragias multifocais na mucosa vesical.

Fig.3. Hemangiossarcoma da bexiga. A luz vesical está preenchida por uma massa exofítica amarela e necrótica (espécime fixado em formol), na forma clínica de hematúria enzoótica bovina.

Fig.5. Metástase de hemangiossarcoma em linfonodo da cavidade pélvica, na forma clínica de hematúria enzoótica bovina. Observam-se áreas branco-amareladas extensas que obliteram parcialmente a arquitetura nodal de um linfonodo da cavidade pélvica (espécime fixado em formol).

Fig.2. Nódulos pálidos e pequenos nódulos vermelhos multifocais na mucosa da bexiga, com estroma mixóide/carcinoma de células de transição/hemangioma, na forma clínica de carcinoma de células escamosas no trato alimentar superior de bovino.

Fig.4. Carcinoma de células de transição na bexiga de bovino, na forma clínica de hematúria enzoótica. Massa tumoral endofítica, com a superfície extensamente ulcerada, recoberta por fibrina, localizada no trígono, e úlceras menores localizadas no corpo da bexiga.

Fig.6. Urocistite polipóide, na forma clínica de hematúria enzoótica bovina. Há projeções multifocais, alongadas e de consistência gelatinosa na mucosa vesical, adjacente a um hemangiossarcoma não vizualizado na foto. 
arregenerativa de intensidade variada. Nos animais anêmicos, o número de eritrócitos variou de 2,1 a 4,8 $\times 10^{6}$ \% $\mathrm{mm}^{3}$ de sangue (VR: 5,0-10,0 $\times 10^{6} / \mathrm{mm}^{3}$ ); o hematócrito de $10-23 \%$ (VR: $24-46 \%$ ) e o VCM de 47,6 a $56,4 \mathrm{fl}$ (VR: 40,0-60,0 fl). Onze bovinos não demonstraram alterações no leucograma e quatro tiveram alterações. Três destes tiveram desvio à esquerda regenerativo (dois com linfopenia concomitante) e um leucocitose por neutrofilia.

\section{Achados macroscópicos}

De um total de 46 casos de CCEs no TAS em que as bexigas foram avaliadas, $16(34,78 \%)$ apresentavam alterações macroscópicas. Em ordem decrescente de freqüência, estas alterações se caracterizavam por nódulos vermelhos elevados (9/46), variando de $0,1 \mathrm{a} 1,0 \mathrm{~cm}$ de diâmetro, na grande maioria múltiplos e distribuídos aleatoriamente pela mucosa vesical; hemorragia (6/46); nódulos pálidos (4/46); edema (3/46), papilomas (2/46) e urina vermelha (2/46, Fig.1). Muitas dessas alterações ocorriam numa mesma bexiga. Nos bovinos em que a urina estava vermelha, um sedimento vermelho-escuro aparecia após a centrifugação; microscopicamente esse sedimento correspondia a eritrócitos.

O bovino que apresentou sinais clínicos das duas formas crônicas da intoxicação tinha uma úlcera focalmente extensa na orofaringe; na superfície de corte, a úlcera aparecia como extensa área de necrose; numerosos nódulos pálidos, alguns com pontos vermelhos eram observados na superfície da bexiga (Fig.2).

Os achados macroscópicos observados no trato urinário dos 11 bovinos com HEB foram: nódulos vesicais multifocais vermelho-escuros ou vermelho-claros (4/11); neoplasmas vesicais focalmente extensos, com alteração da forma normal do órgão (5/11); urina vermelha (4/11; dois casos com coágulos de sangue aderidos à mucosa); hidronefrose com hidroureter (3/11); papilomas vesicais

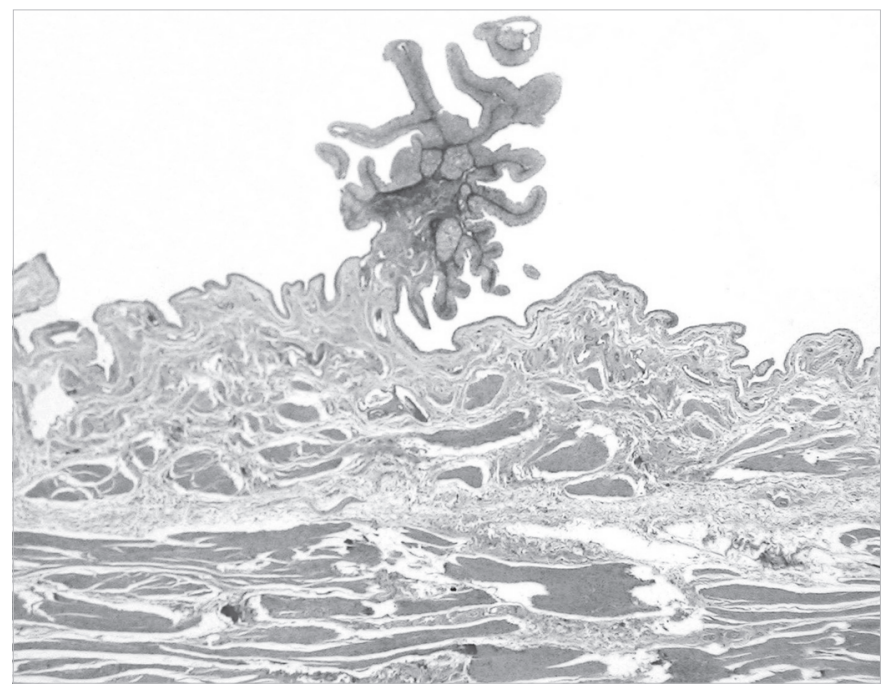

Fig.7. Papiloma na mucosa da bexiga, na forma clínica de carcinomas de células escamosas no trato alimentar superior de bovino. HE, obj.2,5x.
(2/11); hemorragias multifocais $(1 / 11)$, pielonefrite $(1 / 11)$ e ruptura da bexiga (1/11).

Os neoplasmas que ocupavam extensas áreas eram formados por crescimentos exofíticos com superfície irregular, branco-amarelada (Fig.3) ou eram endofíticos, com espessamento da parede da bexiga e superfície extensamente ulcerada recoberta por fibrina (Fig.4). Esses neoplasmas geralmente se localizavam na região do trígono vesical. Em um dos casos, os linfonodos da cavidade pélvica estavam aumentados, com áreas multifocais firmes branco-amareladas (Fig.5). Em outro bovino, havia numerosas projeções, longas e finas com consistência gelatinosa (cistite polipóide), agrupadas em áreas multifocais do neoplasma (Fig.6).

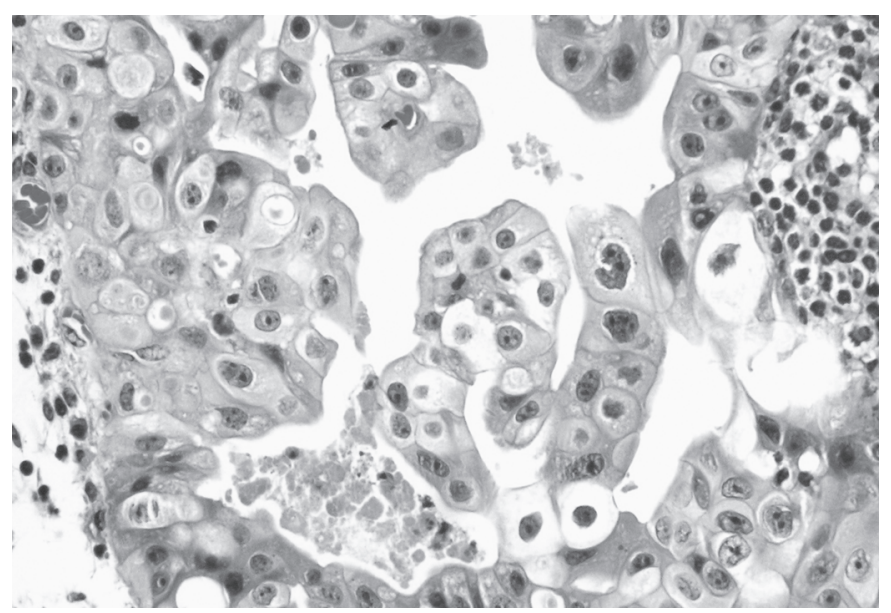

Fig.8. Carcinoma de células de transição na bexiga de bovino, na forma clínica de carcinomas de células escamosas no trato alimentar superior. Células neoplásicas acentuadamente pleomórficas, com núcleos grandes ou pequenos, de formas variadas com um ou dois nucléolos evidentes e citoplasma eosinofílico abundante, às vezes vacuolizado. HE, obj.40x.

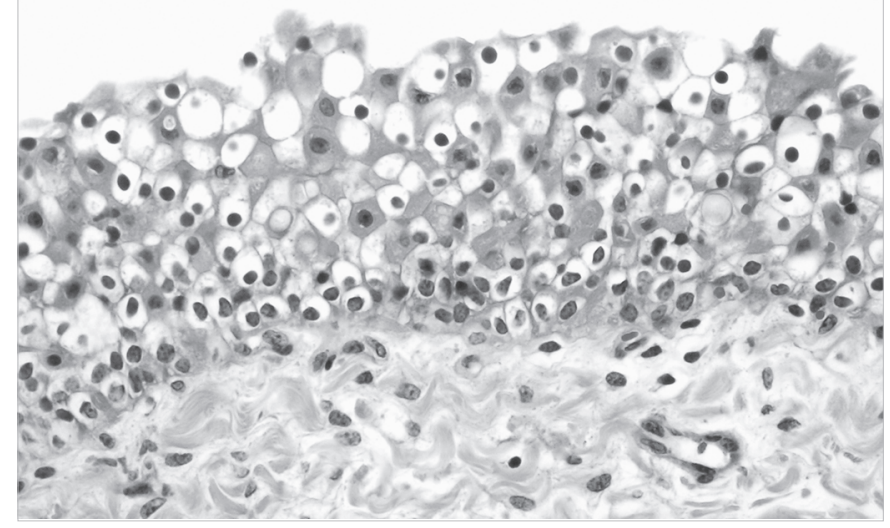

Fig.9. Displasia na mucosa da bexiga de bovino, naforma clínica de carcinomas de células escamosas no trato alimentar superior. Há aumento do número de camadas e perda da orientação das células uroteliais, com vacuolização citoplasmática em algumas delas e núcleos de tamanhos e padrões de cromatina diferentes. HE, obj.20x. 


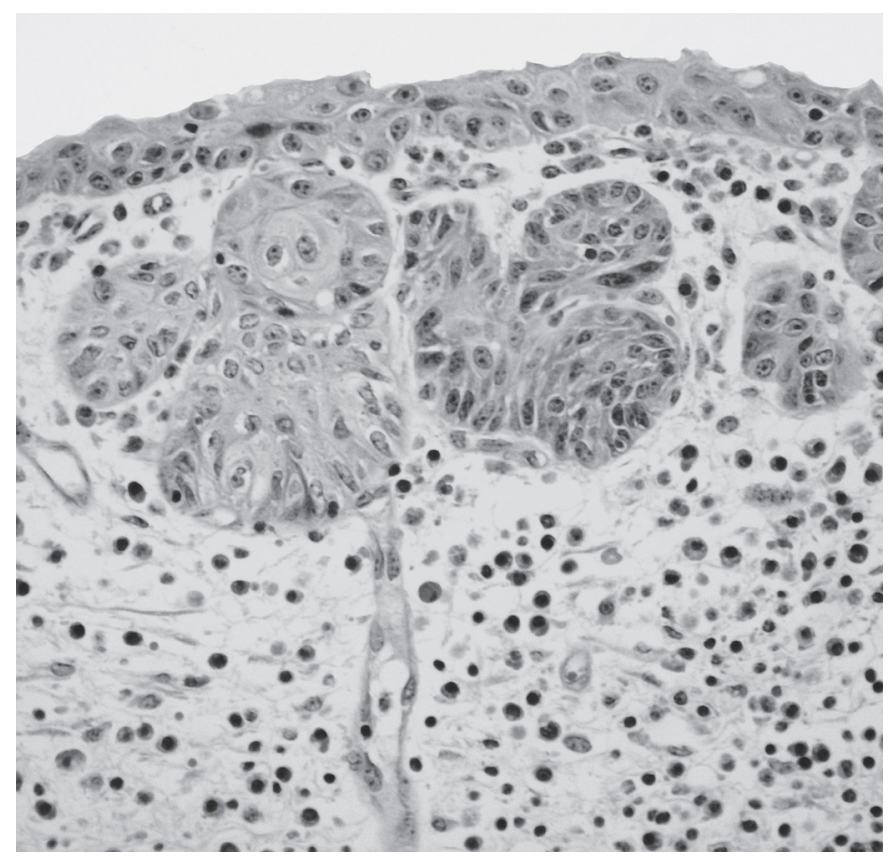

Fig.10. Ninhos de Brunn na lâmina própria da bexiga de bovino, na forma clínica de carcinomas de células escamosas no trato alimentar superior. Há edema acentuado e infiltrado inflamatório linfoplasmocítico moderado. HE, obj.20x.

\section{Achados histopatológicos}

Nas bexigas dos 46 bovinos com CCEs no TAS, 44 $(95,65 \%)$ apresentaram 22 tipos de alterações diferentes. Dessas 22, cinco eram alterações neoplásicas (três neoplasmas benignos e dois malignos) e 17 eram alterações não-neoplásicas. Das alterações não-neoplásicas, seis foram enquadradas em alterações epiteliais não-neoplásicas, seis em alterações gerais na lâmina própria e cinco em alterações somente inflamatórias.

Os neoplasmas benignos observados foram classificados em mesenquimais (um fibroma e quatro hemangiomas) ou epiteliais (papilomas em três bovinos [Fig.7]). Dentre os neoplasmas malignos, havia um de origem mesenquimal (hemangiossarcoma) e um epitelial (carcinoma de células de transição [CCT] em dois bovinos [Fig.8]).

Em três casos houve mais de um tipo de neoplasma nas seguintes combinações: hemangioma e CCT; hemangioma, hemangiossarcoma e papiloma; papiloma e CCT.

As alterações epiteliais não-neoplásicas observadas nas bexigas dos bovinos com CCEs no TAS foram, em ordem decrescente de freqüência, displasia (17/46; Fig.9), caracterizada por vacuolização citoplasmática, proliferação e perda da orientação das células nas camadas do urotélio, com núcleos de tamanhos e padrões de cromatina diferentes; vacúolos intraepiteliais no urotélio (10/46); ninhos de Brunn (8/46), formados por células do epitélio de transição, agrupadas em ninhos na lâmina própria, com ou sem ligação com a superfície epitelial (Fig.10); hiperplasia (3/46), caracterizada por mais de sete camadas de células epiteliais em áreas focalmente extensas do urotélio, cistite cística (2/46), caracterizada pela formação de estruturas císticas na lâmina própria (Fig.11), revestidas por epitélio de transição, algumas contendo substância eosinofílica amorfa intraluminal PAS-positiva e cistite polipóide (1/46), quando havia edema na lâmina própria, causando a formação de pólipos, cobertos por urotélio normal.

As alterações gerais na lâmina própria foram: dilatação de vasos linfáticos (18/46), alguns acompanhados de infiltrado inflamatório perivascular; tumefação de células endoteliais; hemorragias na lâmina própria (8/46; sendo que 5/8 tinham diapedese de eritrócitos pelo urotélio; Fig.12); edema (6/46); estroma mixóide (4/46), quando havia áreas multifocais de tecido conjuntivo frouxo com produção de pequena quantidade de substância amorfa levemente basofílica (Fig.13), azul alciano-positiva e formação focal de tecido fibrovascular (1/46).

As alterações somente inflamatórias consistiam de infiltrado linfoistioplasmocítico (41/46); folículos linfóides (16/ 46) organizados, muitas vezes, ao redor de pequenos vasos; aumento leve nos mastócitos da lâmina própria (5/ 46); cistite fibrinonecrossupurativa (1/46) e cistite histiocítica crônica com fibrose (1/46).

Apenas em dois bovinos não foram observadas alterações histopatológicas na bexiga e em 36 casos, mais de uma alteração histopatológica foi observada.

Nas bexigas de 11 bovinos com HEB foi observado um total de 19 tipos de alterações, em 10 bovinos foram encontradas alterações múltiplas numa mesma bexiga. Das 19 alterações observadas, cinco eram alterações neoplásicas e 14 eram não-neoplásicas (nove classificadas como alterações epiteliais não-neoplásicas, três como alterações gerais na lâmina própria e duas como alterações somente inflamatórias).

Dentre os neoplasmas benignos, dois eram de origem epitelial (papilomas) e três de origem mesenquimal (hemangiomas capilares e/ou cavernosos).

Dentre os neoplasmas malignos, observaram-se três de origem epitelial (dois carcinomas de células escamosas [CCE] e um carcinoma de células de transição [CCT]) e cinco de origem mesenquimal (hemangiossarcomas). Quanto ao comportamento biológico, $38,46 \%$ dos neoplasmas eram benignos e $61,53 \%$ eram malignos. Em dois bovinos havia metástases de CCT (Fig.14) ou de hemangiossarcoma para linfonodos regionais. Em três bovinos havia mais de um tipo de neoplasma: hemangioma e hemangiossarcoma (2/3) ou papiloma e hemangiossarcoma (1/3). Em apenas um dos casos não houve a formação de neoplasmas.

A maioria das alterações não-neoplásicas estava relacionada ao epitélio de transição. Esse grupo continha hiperplasia (5/11); displasia (3/11); ninhos de Brunn (6/11); vacúolos intraepiteliais (5/11); cistite polipóide (2/11; Fig.15); cistite cística (2/11); metaplasia glandular (3/11), quando células isoladas ou grupos de células no urotélio e na lâmina própria produziam muco PAS e azul alciano-positivo; cistite glandular (1/11), quando as células referidas anteri- 


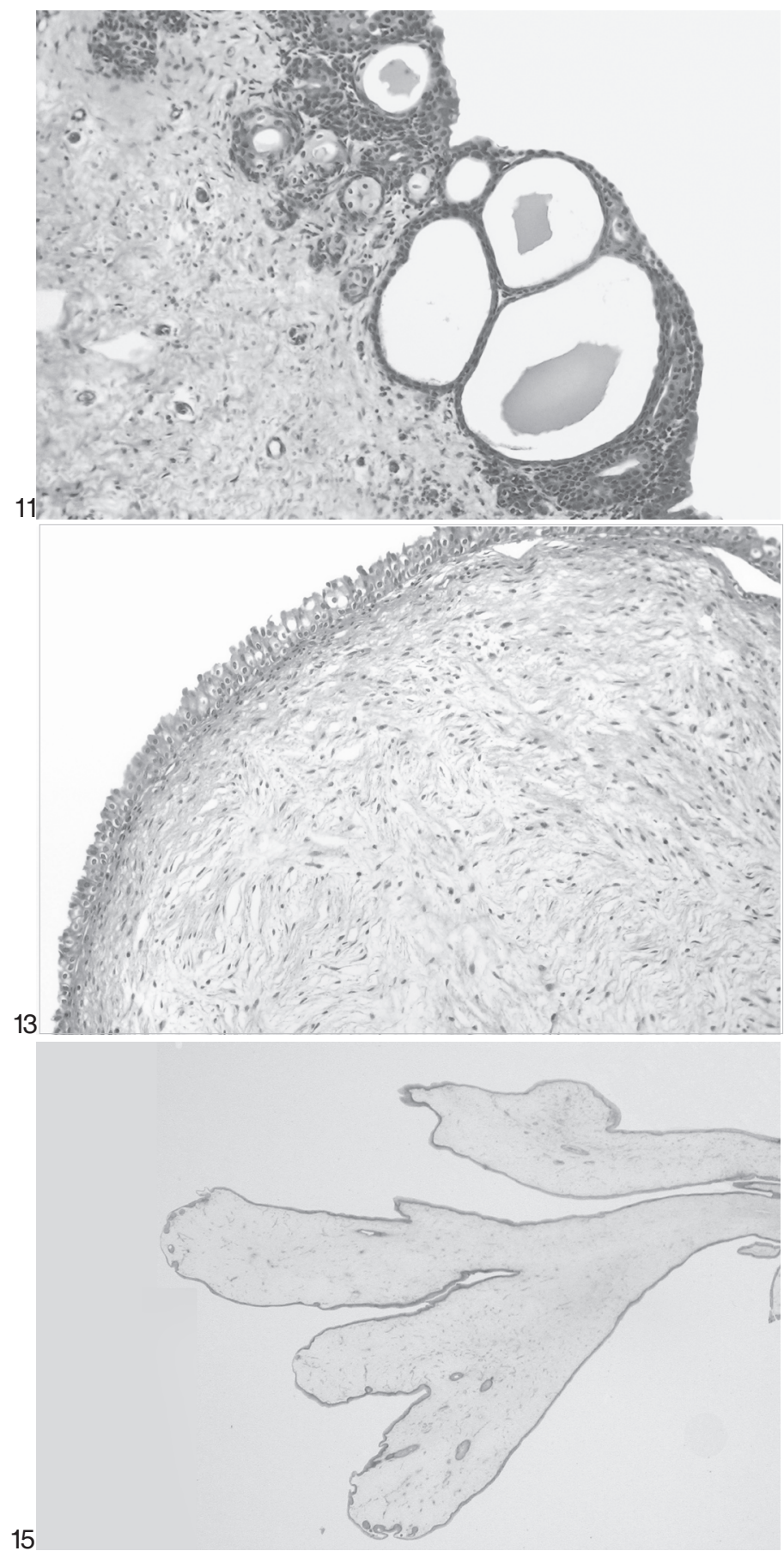

Fig.11. Urocistite cística, na forma clínica de carcinomas de células escamosas no trato alimentar superior de bovino. Proliferação de células epiteliais formando cistos, contendo material amorfo eosinofílico intraluminal. Observam-se ainda ninhos de Brunn na lâmina própria (setas). HE, obj.10x.

Fig.13. Proliferação de células fusiformes ou estreladas arranjadas frouxamente e envoltas por matriz eosinofílica, formando um nódulo bem delimitado na lâmina própria de estroma mixóide na bexiga, na forma clínica de carcinomas de células escamosas no trato alimentar superior de bovino. HE, obj.4x.

Fig.15. Urocistite polipóide, na forma clínica de hematúria enzoótica bovina. Observam-se projeções recobertas por epitélio de transição, com edema acentuado na lâmina própria. HE, obj.2,5x.

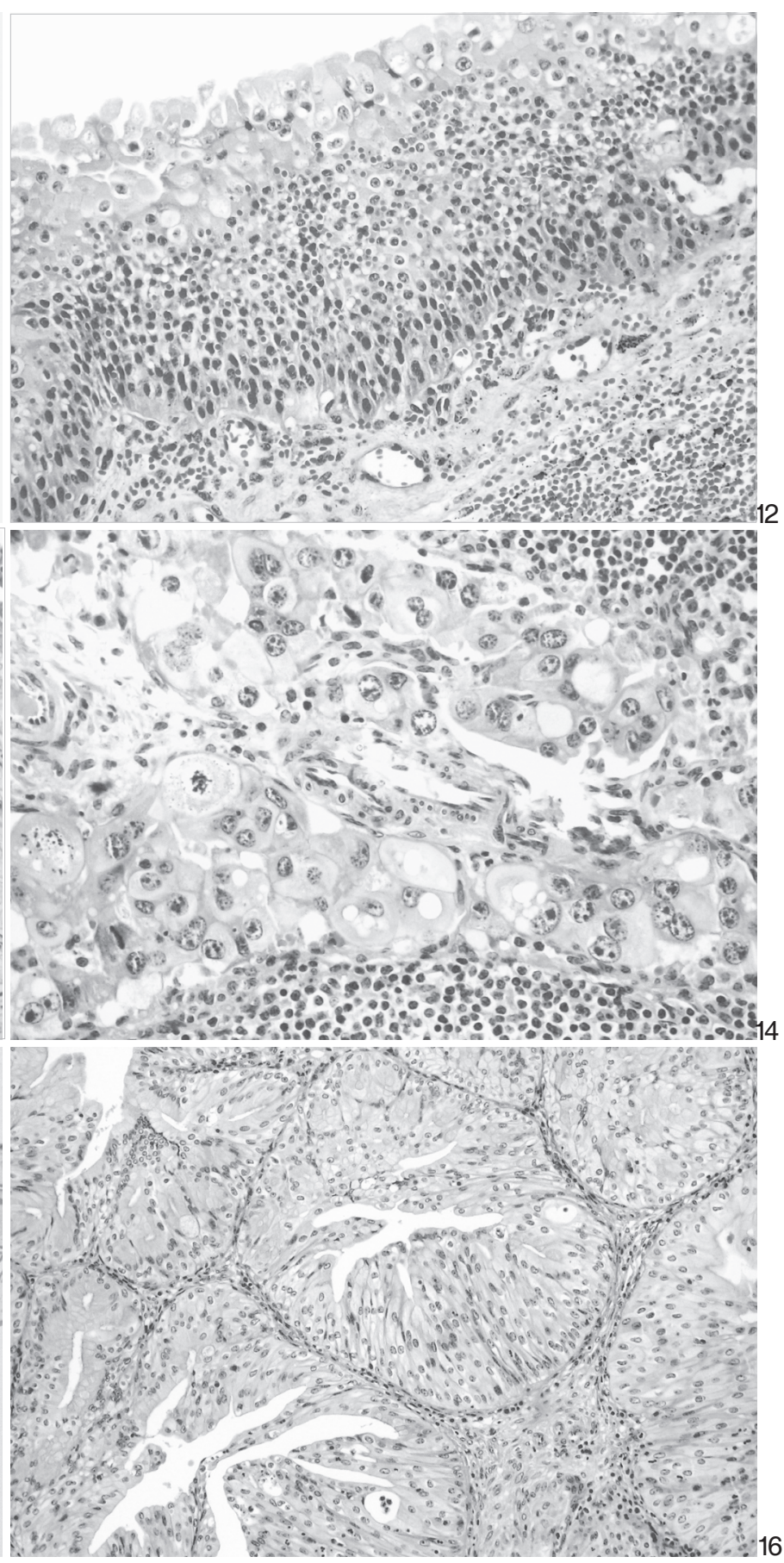

Fig.12. Diapedese de eritrócitos pelo urotélio hiperplásico. Há hemorragia acentuada na lâmina própria, na forma clínica de carcinomas de células escamosas no trato alimentar superior de bovino. HE, obj.20x.

Fig.14. Metástase de carcinoma de células de transição em linfonodo renal, na forma clínica de hematúria enzoótica bovina. Há células epiteliais neoplásicas nos seios trabeculares do linfonodo. As células neoplásicas são acentuadamente pleomórficas e observam-se mitoses. HE, obj.40x.

Fig.16. Urocistite glandular, na forma clínica de hematúria enzoótica bovina. Proliferação de células epiteliais formando estruturas glandulares na lâmina própria. HE, obj.10x. 
ormente formavam estruturas semelhantes a glândulas que invadiam a lâmina própria (Fig.16); e metaplasia escamosa (1/11), caracterizada pela proliferação de células bem diferenciadas do urotélio, as quais sofriam ceratinização à medida que se aproximavam da luz vesical.

As alterações na lâmina própria, observadas nos 11 casos de HEB perfizeram 3/14 alterações não-neoplásicas e foram: dilatação de vasos linfáticos (6/11), associada ou não a infiltrado inflamatório adjacente; hemorragia (6/ 11; $3 / 6$ com diapedese de eritrócitos pelo urotélio) e edema leve a acentuado na lâmina própria (6/11).

Foram observados dois tipos de alterações somente inflamatórias (2/14): formação de folículos linfóides (distribuídos multifocalmente na lâmina própria) em seis dos casos e infiltrado inflamatório (composto por linfócitos, plasmócitos e histiócitos) em 10 dos casos estudados.

No rim de um bovino observaram-se alterações histológicas consistentes com pielonefrite.

\section{DISCUSSÃO}

Os sinais clínicos observados nos bovinos com CCEs no TAS estão de acordo com os descritos na literatura (Döbereiner et al. 1967, Tokarnia et al. 1969, Tokarnia et al. 2000) e já foram relatados ou discutidos em outros estudos, que utilizaram bovinos da mesma região (Souto 2006a, Masuda 2007).

Em relação à HEB, a hematúria foi o sinal clínico observado em todos os casos e o parâmetro utilizado para a inclusão dos nove casos clínicos de HEB do presente estudo. Esse achado clínico é considerado o sinal cardinal dessa doença (Pamukcu et al. 1976); esse sinal inicia com micro-hematúria, condição em que os eritrócitos na urina são observados apenas microscopicamente (Rosenberger 1971). Semanas ou meses após, já se pode observar macro-hematúria, onde a urina fica tingida de vermelho e, após a centrifugação, ocorre a formação de um sedimento de eritrócitos (Rosenberger 1971). A hematúria pode ser intermitente ou contínua (Gava 1993), durando poucas semanas a meses (Pamukcu et al. 1976).

Apesar da marcada sobreposição das lesões vesicais nas duas formas crônicas da intoxicação, que será discutida mais adiante, clinicamente apenas um bovino apresentou sinais clínicos das duas formas. Este caso foi incluído, em todos os parâmetros avaliados, como um caso de CCE no TAS, pois a lesão mais grave observada nesse bovino se localizava no TAS, indo ao encontro do que já foi previamente descrito (Döbereiner et al. 1967, Tokarnia et al. 1969).

Através dos hemogramas, foi observado que 33,33\% dos bovinos com CCEs no TAS apresentaram anemia arregenerativa leve. Em 5/10 casos com anemia, observaram-se morfologicamente hemorragia na lâmina própria e/ ou hemangiomas na bexiga, o que talvez tenha permitido a perda de sangue pela urina. Os resultados obtidos no eritrograma dos bovinos deste estudo serão discutidos posteriormente. Apenas um trabalho experimental, dentre os consultados, relata as alterações hematológicas relacio- nadas a essa forma de intoxicação crônica por Pteridium aquilinum, sendo que as alterações são descritas apenas no leucograma (Campo et al. 1994). Linfopenia é um achado hematológico importante em bovinos cronicamente e experimentalmente intoxicados (Campo et al. 1994), porém foi observado em apenas um bovino deste estudo. É importante salientar que neste estudo os hemogramas foram realizados com o objetivo de determinar o perfil hematológico da fase terminal da intoxicação e talvez isso explique as diferenças de alguns resultados aqui obtidos dos descritos na literatura, onde a doença foi reproduzida experimentalmente e os animais foram acompanhados periodicamente por longos períodos (Campo et al. 1994). Todos os bovinos deste estudo que apresentaram desvio a esquerda tinham CCEs ulcerados no TAS e/ou abscessos adjacentes aos CCEs.

Nos bovinos com HEB aqui estudados, $66,67 \%$ apresentaram anemia arregenerativa normocítica. As alterações no eritrograma observadas em bovinos com HEB incluem a diminuição dos valores do hematócrito $(\mathrm{Ht})$, hemoglobina, número de eritrócitos e volume corpuscular médio (VCM) (Sánchez-Villalobos et al. 2004). No presente estudo houve diminuição no número de eritrócitos e no valor do $\mathrm{Ht}$, porém não houve alteração no $\mathrm{VCM}$, caracterizando morfologicamente a anemia como normocítica e, conseqüentemente como arregenerativa. A anemia observada em bovinos com HEB tem sido relacionada à contínua perda de sangue pela urina (Singh et al. 1973), no entanto as anemias hemorrágicas se caracterizam por serem bastante regenerativas (Fighera 2001), característica essa que não foi observada no presente estudo, nas duas formas crônicas da intoxicação. A presença de hematúria evidencia a perda de sangue, que com certeza estava ocorrendo nestes bovinos, porém outro fator interferiu, impedindo a regeneração do tecido eritróide, regeneração esta esperada nas anemias por perda de sangue. Um tipo de anemia, denominada anemia por doença crônica, associada à redução na proliferação eritróide e à utilização inadequada do ferro é descrito (Cotran et al. 2000). A anemia por doença crônica ocorre associada a doenças crônicas infecciosas, inflamatórias, traumáticas e a neoplasmas (Lee et al. 1998) e caracteriza-se por ser arregenerativa leve (Meuten 2002, Thrall 2006). Talvez esse tipo de anemia possa explicar os resultados aqui obtidos. Outra hipótese é que o princípio ativo da planta estivesse agindo diretamente sobre a medula óssea, já que na forma aguda da intoxicação ocorre rarefação do tecido hematopoético (Tokarnia et al. 2000). Porém, alterações na hematopoese nas formas crônicas da intoxicação não foram descritas na literatura consultada.

Em cinco bovinos com HEB não houve anemia, resultado também descrito na literatura, em bovinos com microhematúria (Falbo et al. 2005). A ausência de anemia em animais que já desenvolveram macro-hematúria, como vista em $5 / 15$ casos deste estudo, não foi relatada nos trabalhos revisados. As alterações no leucograma ocor- 
reram em apenas quatro bovinos com HEB. Alterações na contagem total de leucócitos não foram observadas por alguns autores em bovinos com HEB (Singh et al. 1973, Falbo et al. 2005), porém outros autores descreveram leucopenia como um achado freqüente (Hopkins 1986, Marrero et al. 2001). Nos bovinos do presente estudo não foi observada leucopenia, e sim leucocitose por neutrofilia, sendo que nesses casos havia ulceração epitelial vesical, cistite ou pielonefrite, o que explica esse achado. Em apenas dois bovinos com HEB observou-se linfopenia, sendo este um achado importante em bovinos de um estudo experimental (Campo et al. 1992).

$\mathrm{Na}$ avaliação macroscópica, 34,78\% das bexigas de bovinos com CCEs no TAS apresentavam lesões macroscópicas na mucosa vesical, muito semelhantes às observadas nos casos de HEB. Este achado está de acordo com o relatado anteriormente, onde $30 \%$ dos casos de CCE no TAS tinham também neoplasmas na bexiga (Jarrett et al. 1978). A coexistência de lesões no TAS e na bexiga ocorre nos bovinos, e a lesão mais acentuada, responsável pelo surgimento dos sinais clínicos, é o que determina o quadro clínico apresentado pelo animal (Tokarnia et al. 1969). Apesar de no presente estudo apenas um bovino apresentar sinais clínicos das duas formas da intoxicação crônica, no exame macroscópico, mais dois bovinos tinham urina vermelha na bexiga, que depois de centrifugada revelou um sedimento de eritrócitos, o que caracteriza a hematúria.

As diversas alterações macroscópicas observadas nas bexigas de bovinos com HEB estão de acordo com o descrito na literatura (Pamukcu et al. 1967, Carvalho et al. 2006). Hidronefrose com hidroureter, ruptura da bexiga e pielonefrite, como vistas em poucos casos, são alterações que ocorrem como complicações graves dos neoplasmas vesicais, causadas por obstrução do fluxo urinário e infecção bacteriana ascendente (Rosenberger 1971) e são pouco observadas na HEB (Tokarnia et al. 2000). Em apenas um dos casos, observaram-se metástases macroscópicas para os linfonodos regionais. Metástases são descritas eventualmente em bovinos com HEB e geralmente ocorrem para os linfonodos regionais e órgãos adjacentes ao neoplasma (Tokarnia et al. 2000).

Histologicamente, 44 bexigas de 46 casos com CCEs no TAS apresentaram algum tipo de alteração. Este resultado é interessante quando comparado com as alterações observadas macroscopicamente (apenas 16/46 bexigas). As lesões vesicais apenas microscopicamente visíveis, observadas neste trabalho, especialmente as alterações não-neoplásicas, não foram descritas em casos de CCE no TAS na literatura consultada. Os neoplasmas observados neste estudo, nos casos de CCEs no TAS, foram benignos ou malignos, epiteliais ou mesenquimais. Hemangiomas, fibromas, carcinomas de células de transição e adenocarcinomas também foram observados nas bexigas de bovinos com CCEs no TAS (Jarrett et al. 1978). As alterações epiteliais não-neoplásicas observadas nos bovinos com CCEs no TAS englobaram uma série de al- terações histológicas, sendo muitas delas displásicas. Displasias são consideradas alterações pré-neoplásicas, porém, nem todas elas evoluem para uma neoplasia (Cullen et al. 2002).

As alterações na lâmina própria e as inflamatórias, conforme observadas neste estudo, podem anteceder ou ocorrer concomitantemente aos neoplasmas vesicais (Meuten 2002). Alterações vasculares descritas em casos de HEB variam desde a proliferação de vasos sanguíneos bem diferenciados até a proliferação de células endoteliais da íntima das artérias, formando pequenas papilas no lúmen vascular (Peixoto et al. 2003). Vasos sanguíneos alterados provocam hemorragias para a parede ou lúmen vesical e alterações hemangiomatosas podem se desenvolver nessas áreas, ocasionando macrohematúria, que, em alguns casos, ocorre sem o desenvolvimento de lesões neoplásicas (Newman et al. 2007). Neste estudo foram observados casos com macro-hematúria, sem lesões neoplásicas vesicais, tanto nos casos de CCEs no TAS quanto nos casos de HEB.

Aproximadamente $30 \%$ das alterações observadas nas bexigas de bovinos com HEB eram neoplásicas, sendo os neoplasmas mesenquimais mais observados $(61,53 \%)$ que os epiteliais $(38,46 \%)$. Outros estudos descrevem maior quantidade de neoplasmas epiteliais (Carvalho et al. 2006) e de neoplasmas mistos, contendo células mesenquimais e epiteliais num mesmo tumor (Pamucku et al. 1976). Em três casos havia mais de um tipo histológico de neoplasma no mesmo bovino, achado também descrito na literatura (Carvalho et al. 2006). A maioria dos neoplasmas eram malignos $(61,53 \%)$, o que está de acordo com o descrito na literatura (Carvalho et al. 2006). Em dois casos, observou-se metástase para os linfonodos regionais, sendo que em um deles a metástase foi detectada somente na avaliação microscópica. Por razões ainda não determinadas, metástases raramente ocorrem em casos de HEB, mesmo naqueles em que os neoplasmas são acentuadamente pleomórficos e com caráter invasivo (Peixoto et al. 2003).

Aproximadamente $70 \%$ das alterações vesicais observadas em bovinos com HEB eram não-neoplásicas e, apesar de não terem a mesma prevalência aqui observada, foram também descritas por outros autores (Peixoto et al. 2003, Sardon et al. 2005, Carvalho et al. 2006). Essas alterações muitas vezes estão associadas a neoplasias vesicais e também contribuem para o diagnóstico histológico da doença (Borzacchiello \& Roperto 2008). As alterações epiteliais foram as mais prevalentes das alterações não-neoplásicas observadas nos casos de HEB. Esses achados são relevantes, pois podem significar alterações iniciais, as quais antecedem alterações neoplásicas. Neoplasmas epiteliais parecem se desenvolver a partir de alterações hiperplásicas e metaplásicas (escamosas e glandulares) no urotélio, freqüentemente acompanhadas de alterações vasculares (Newman et al. 2007). Os carcinógenos químicos em geral, induzem a formação de neoplasmas após uma série de eventos ce- 
lulares e histológicos, que iniciam com hiperplasia focal do urotélio e metaplasia escamosa, as quais evoluem para displasias, que por sua vez se transformam em neoplasias (Meuten 2002).

As mais diversas alterações vesicais aqui observadas talvez tenham sido induzidas pelos princípios tóxicos da planta, os quais são eliminados de forma ativa pela urina (Lioi et al. 2004). A coexistência de lesões vesicais e no TAS de bovinos intoxicados cronicamente por samambaia, como vista neste estudo, possivelmente tenha relação com o pH alcalino dessas regiões. Em pH alcalino, o ptaquilosídeo é convertido a uma dienona instável que é subseqüentemente carcinógena/mutágena (Fenwick 1988). A avaliação de uma possível participação do papilomavírus bovino tipo 2 (BPV-2) na gênese das lesões vesicais, conforme relatado na literatura (Borzacchiello \& Roperto 2008), não foi alvo deste estudo.

A técnica de imuno-histoquímica mostrou ser eficiente no diagnóstico de neoplasmas pouco diferenciados. A utilização dos anticorpos anti-pancitoceratina e antivimentina possibilitaram a diferenciação entre células neoplásicas epiteliais e mesenquimais, respectivamente. Em dois casos, a utilização de anticorpo anti-fator de von Willebrand, permitiu o diagnóstico de dois hemangiossarcomas, que tinham sido previamente classificados como sarcomas indiferenciados. Nos dois neoplasmas obtevese a imunomarcação de poucas células neoplásicas.

\section{CONCLUSÕES}

Cada uma das formas crônicas da intoxicação por Pteridium aquilinum apresenta sinais clínicos bastante definidos e a sobreposição dos sinais foi raramente observada neste estudo.

A principal alteração hematológica observada nas formas crônicas da intoxicação foi anemia arregenerativa; alterações do leucograma ocorreram em alguns casos, mas linfopenia foi um achado infreqüente em ambas as formas de intoxicação.

A ocorrência de lesões vesicais concomitantemente com a forma crônica de CCEs no TAS em bovinos é comum e as lesões macroscópicas e histopatológicas observadas nas bexigas desses bovinos são idênticas às encontradas nos casos de HEB.

Além das alterações neoplásicas, diversas alterações não-neoplásicas são observadas nas bexigas dos bovinos, tanto com CCEs no TAS quanto com HEB, demonstrando que essas alterações são comumente observadas na intoxicação crônica por samambaia e devem ser consideradas no diagnóstico da doença.

Agradecimentos.- Ao Conselho Nacional de Desenvolvimento Científico e Tecnológico (CNPq) pelo suporte financeiro (478692/2007-2). G.D. Kommers é bolsista de produtividade do CNPq.

\section{REFERÊNCIAS}

Borzacchiello G. \& Roperto F. 2008. Bovine papillomaviruses, papillomas and cancer in cattle. Vet. Res. 39:45.
Campo M.S., Jarrett W.F.H., Barron R., Neil B.W.O. \& Smith K.T. 1992. Association of bovine papillomavirus type 2 and bracken fern with bladder cancer in cattle. Cancer Res. 52:6898-6904.

Campo M.S., Neil B.W.O., Barron R.J. \& Jarrett W.F.H. 1994. Experimental reproduction of the papiloma-carcinoma complex of the alimentary canal in cattle. Carcinogenesis 15:1597-1601.

Carvalho T., Pinto C. \& Peleteiro M.C. 2006. Urinary bladder lesions in Bovine Enzootic Haematuria. J. Comp. Pathol.134:336-346.

Cotran R.S., Kumar V. \& Collins T. 2000. Neoplasia, p.233-295. In: Ibid. (Ed.), Robbins' Patologia Estrutural e Funcional. Guanabara Koogan, Rio de Janeiro. 1251p.

Cullen J.M., Page R. \& Misdorp W. 2002. An overview of cancer pathogenesis, diagnosis, and management, p.3-44. In: Meuten D.J. (Ed.), Tumors in Domestic Animals. Iowa State Press, Ames. 788p.

Döbereiner J., Tokarnia C.H. \& Canella C.F.C. 1967. Ocorrência da hematúria enzoótica e de carcinomas epidermóides no trato digestivo superior em bovinos no Brasil. Pesq. Agropec. Bras. 2:489-504.

Falbo M.K., Reis A.C.F., Balarin M.R.S., Bracarense A.P.F.R.L., Araújo J.P., Okano W. \& Sandini I.E. 2005. Alterações hematológicas, bioquímicas, urinárias e histopatológicas na intoxicação natural em bovinos pela samambaia Pteridium aquilinum (L.) Kuhn. Semina, Ciências Agrárias 26:547-558.

Fenwick G.R. 1988. Bracken (Pteridium aquilinum): Toxic effects and toxic constituents. J. Sci. Food Agric. 46:147-173.

Fighera R.A. 2001. Anemia em Medicina Veterinária. Editora Pallotti, Santa Maria. 214p.

Gava A. 1993. Intoxicações por plantas de ação anti-hematopoética e mutagênica, p.247-258. In: Riet-Correa F., Méndez M.D.C. \& Schild A.L. (Eds), Intoxicações por Plantas e Micotoxicoses em Animais Domésticos. Hemisfério Sul, Pelotas. 340p.

Gava A., Schild A.L. \& Riet-correa F. 2002. Bracken fern (Pteridium aquilinum) poisoning in cattle in southern Brazil. Vet. Human Toxicol. 44:362-365

Hirono I., Fushimi K., Mori K., Miwa T. \& Haga M. 1973. Comparative study of carcinogenic activity in each part of bracken. J. Natl Cancer Inst. 50:1367-1371.

Hopkins N.C.G. 1986. Aetiology of enzootic haematuria. Vet. Rec. 118:715-717.

Jain N.C. 1986. Cattle: Normal hematology with comments on response to disease, p.178-207. In: Jain N.C. (Ed.), Schalm's Veterinary Hematology. Lea and Febiger, Philadelphia. 1221p.

Jarrett W.F.H., McNeil P.E., Grimshaw W.T.R., Selman I.E. \& McIntyre. 1978. High incidence area of cattle cancer with a possible interaction between an environmental carcinogen and a papilloma virus. Nature 274(20):215-217.

Lee G.R., Bithell T.C., Foerster J., Athens J.W. \& Lukens J.N. 1998. Wintrobe Hematologia Clínica. Ed. Manole, São Paulo. 1424p.

Lioi M.B., Barbieri R., Borzacchiello G., Dezzi S., Roperto S., Santoro A., Russo V. \& Roperto F. 2004. Chromosome aberrations in cattle with chronic enzootic haematuria. J. Comp. Pathol. 131:233-236.

Marrero E., Bulnes C., Sánchez L.M. \& Palenzuela I. 2001. Pteridium aquilinum (Bracken fern) toxicity in cattle in the Humid Chaco of Tarija, Bolivia. Vet. Human Toxicol. 43:156-158.

Masuda E.K. 2007. Morfologia e imunoistoquímica dos carcinomas de células escamosas alimentares associados ao consumo de Pteridium aquilinum em bovinos. Dissertação de Mestrado em Medicina Veterinária, UFSM, Santa Maria, RS. 127p.

Meuten D.J. 2002. Tumors of the urinary system, p.509-573. In: Ibid. (Ed.), Tumors in Domestic Animals. lowa State Press, Ames. 788p.

Murphy W.M., Grignon D.J. \& Perlan E.J. 2004. Tumors of the kidney, bladder, and related urinary structures (AFIP atlas of tumor pathology). Armed Forces Institute of Pathology, Philadelphia. 394p.

Newman S.J., Confer A.W. \& Panciera R.J. 2007. Urinary system, p.613- 
691. In: McGavin M.D. \& Zachary J.F. (Eds), Pathologic Basis of Veterinary Disease. Mosby Elsevier, St Louis. 1488p.

Pamukcu A.M., Göksoy S.K. \& Price J.M. 1967. Urinary bladder neoplasms induced by feeding bracken fern (Pteris aquilina) to cows. Cancer Research 27:917-924.

Pamukcu A.M., Price J.M. \& Bryan G.T. 1976. Naturally occurring and bracken-fern-induced bovine urinary bladder tumors-clinical and morphological characteristics. Vet. Pathol. 13:110-122.

Peixoto P.V., França T.N., Barros C.S.L. \& Tokarnia C.H. 2003. Histopathological aspects of bovine enzootic hematuria in Brazil. Pesq. Vet. Bras. 23:65-81.

Riet-Correa F. \& Medeiros R.M.T. 2001. Intoxicações por plantas em ruminantes no Brasil e no Uruguai: importância econômica, controle e riscos para a saúde pública. Pesq. Vet. Bras. 21:38-42.

Rosenberger G. 1971. Nature, manifestations, cause and control of chronic enzootic haematuria in cattle. Vet. Med. Rev. 2:189-206.

Sánchez-Villalobos A., Alvarado C.M.A., García-Bracho D., PinoRamírez D., Villarroel-Neri R. \& Boscan-Ocando J. 2004. Observaciones clínico patológicas en vacas con hematuria enzoótica bovina. Revta Cient. FCV-LUZ 14:317-323.

Sardon D., Fuente I., Calonge E., Perez-Alenza M.D., Castaño M., Dunner S. \& Peña L. 2005. H-ras immunohistochemical expression and molecular analysis of urinary bladder lesions in grazing adult cattle exposed to bracken fern. J. Comp. Pathol. 132:195-201.
Singh A.K., Joshi H.C. \& Ray S.N. 1973. Studies on bovine haematuria. I. Haematological and biochemical observations on the blood of cattle suffering from haematuria. Indian J. Anim. Sci.. 43:296-299.

Souto M.A.M., Kommers G.D., Barros C.S.L., Piazer J.V.M., Rech R.R., Riet-Correa F. \& Schild A.L. 2006a. Neoplasias do trato alimentar superior de bovinos associadas ao consumo espontâneo de samambaia (Pteridium aquilinum). Pesq.Vet. Bras. 26:112-122.

Souto M.A.M., Kommers G.D., Barros C.S.L., Rech R.R. \& Piazer J.V.M. 2006b. Neoplasmas da bexiga associados à hematúria enzoótica bovina. Ciência Rural 36:1647-1650.

Thrall M.A. 2006. Hematologia e Bioquímica Clínica Veterinária. Roca, São Paulo. 582p.

Tokarnia C.H., Döbereiner J. \& Canella C.F.C. 1969. Ocorrência da hematúria enzoótica e de carcinomas epidermóides no trato digestivo superior em bovinos no Brasil. II. Estudos complementares. Pesq. Agropec. Bras. 4:209-224.

Tokarnia C.H., Döbereiner J. \& Peixoto P.V. 2000. Plantas de ação radiomimética, p.178-187. In: Ibid. (Eds), Plantas Tóxicas do Brasil. Editora Helianthus, Rio de Janeiro. 310p.

Villalobos Salazar J.V., Hernandes H., Meneses A. \& Salazar G. 1999. Factors which may affect ptaquiloside levels in milk: Effects of altitude, bracken fern growth stage, and milk processing. Proceedings of the International Bracken Group Conference - Bracken Fern: Toxicity, biology and control. Manchester, UK, p.68-74. 